\title{
Efectos e impactos ambientales en la producción y aplicación del abono supermagro en el cultivo de sandía
}

\section{Environmental effects and impacts on the production and application of supermagro biofertilizer to grow watermelon}

\author{
Jorge Didier González ${ }^{[1]}$, Juan Diego Mosquera ${ }^{[2]}$ y Armando Torrente Trujillo ${ }^{[3}$
}

\begin{abstract}
Resumen
El propósito de la investigación fue evaluar la producción e impactos ambientales en la aplicación de abonos orgánicos de estilo Supermagro en el cultivo de Sandía (Citrullus lanatus), mediante un diseño completamente al azar con cuatro tratamientos y cuatro repeticiones (supermagro al 10\%, supermagro al 30\%, fertilizante químico - Agrimins y un testigo). La investigación demostró que la aplicación de abono orgánico Supermagro al 30\% influye en las características agronómicas de altura de planta, engrosamiento del tallo y fructificación, por lo que se concluye que el fertilizante Supermagro responde en las etapas fisiológicas del cultivo y presenta potencialidad para ser utilizado en la producción frutícola con un buen manejo agronómico, demostrando impactos ambientales positivos.
\end{abstract}

Palabras clave: Abonos orgánicos; cultivo de Sandía; Impactos ambientales.

\begin{abstract}
The purpose of this paper was to assess the production and environmental impact of the application of supermagro type organic manure in watermelon crops (Citrullus lanatus). We did this through a random design with four treatments and four repetitions (10\% supermagro, $30 \%$ supermagro, Agrimins chemical fertilizer and a control). The study proved that the application of $30 \%$ supermagro biofertilizer affected the agronomic traits associated to plant height, stem thickening and fructification. We therefore conclude that supermagro biofertilizer responds well in the crop's physiological process and could potentially be used in fruit production with good agronomic practices, showing positive environmental impacts.
\end{abstract}

Key words: Organic manure; watermelon crops, environmental impact.

[1] Ingeniero Agrícola. Universidad Surcolombiana - Neiva. Av. Pastrana - Carrera 1. didier585@hotmail.com

[2] Ingeniero Agrícola. Universidad Surcolombiana - Neiva. Av. Pastrana - Carrera 1. juancho3173027460@hotmail.co

[3] Ph.D. Profesor Titular, Universidad Surcolombiana. Armando Torrente Trujillo. armando.torrente@gmail.com.

Recibido: 22 febrero 2015 • Aceptado: 1 mayo 2015 


\section{Introducción}

La producción de alimentos en un mundo globalizado requiere de la mayor eficacia, además de ajustarse a las leyes de inocuidad para lograr que los productos logren la aceptación de los mercados nacionales y mundiales. Los sistemas de agricultura convencional están basados en la aplicación de abonos minerales solubles y en muchos casos no se tienen en cuenta los mecanismos de absorción de la planta, los equilibrios existentes entre ésta y el suelo, ni los bloqueos o sinergias entre los nutrientes; debido a que las plantas sintetizan sus alimentos a partir de elementos químicos que toman del aire, agua y suelo. Existen 60 elementos químicos constituyentes de las plantas, de los cuales 16 son esenciales (García \& Dorronsoro, 1998).

Existen prácticas de fertilización intensiva en busca de obtener los máximos rendimientos, aún a costa de generar consumos de lujo y promover la aparición de plagas, debido a cambios en la composición nutritiva de los tejidos vegetales; con esta práctica también se promueve la degradación ambiental por la lixiviación de nutrientes (Valenzuela et al, 2012), la pérdida de materia orgánica del suelo, erosión y se genera la necesidad de emplear grandes cantidades de herbicidas, fungicidas y plaguicidas.

El uso intensivo e indiscriminado de agroquímicos y fertilizantes de síntesis química con alta concentración de nutrientes en la agricultura ha promovido diversos problemas del orden ambiental, como la contaminación de alimentos, el agua y el suelo, desequilibrios biológicos (eliminación de organismos benéficos, eutrofización y surgimiento de resistencia de patógenos y plagas), y reducción de la diversidad (Garzón y Perdomo, 2013). Por ello la producción agropecuaria tiene unos profundos efectos en el medio ambiente, siendo la principal fuente de contaminación. La contaminación por fertilizantes se produce cuando éstos se utilizan en mayor cantidad de la que pueden absorber los cultivos, o cuando se eliminan por acción del agua o del viento de la superficie del suelo antes de que puedan ser absorbidos (Gonzáles, 2011). Para reducir el impacto de los agroquímicos sobre el ambiente y la calidad de los productos, se recomiendan sistemas de producción orgánica que reduzcan o supriman el uso de fertilizantes, insecticidas, herbicidas, hormonas y reguladores de crecimiento inorgánicos.

La agricultura orgánica controlada es una alternativa para la producción sostenida de alimentos limpios y sanos a bajo costo, puesto que es un sistema de producción en el cual no se utilizan insumos contaminantes nocivos para las plantas, para el ser humano, el agua, el suelo y el medio ambiente. El uso de abonos orgánicos ha cobrado gran importancia por diversas razones (Nieto-Garibay et al., 2002), teniendo como respuesta la mejora en las prácticas agrícolas. Dentro de los abonos orgánicos sobresalen el compost, el vermicompost y la descomposición anaerobia, debido a que sus procesos de elaboración son métodos biológicos que transforman restos orgánicos de distintos materiales en productos relativamente estables (Claassen \& Carey, 2004), pero muchas veces el desconocimiento de los pasos y materiales involucrados en la preparación de las mezclas conducen al agricultor a tomar decisiones equivocadas.

Por ello la tendencia actual, es la investigación de nuevos procesos de fertilización orgánica para el mejoramiento del suelo y el crecimiento de las plantas, como la búsqueda de nuevos materiales o mezclas que además de proporcionar mejores condiciones de crecimiento al cultivo, disminuyan el impacto ambiental, mantengan el equilibrio general y el flujo de nutrientes, buscando máximas eficiencias, reduciendo costos y haciendo el manejo adecuado de los desechos. Se hace necesaria la caracterización de las propiedades de los abonos orgánicos para un uso efectivo en áreas de cultivo e identificar los efectos sobre el suelo y las plantas de manera que contribuyan al efectivo y sano crecimiento y desarrollo de las plantaciones y a su vez al mejoramiento de los suelos y el medio ambiente.

El propósito de la investigación es evaluar la respuesta del cultivo de sandía a la aplicación del abono orgánico Supermagro, como los impactos ambientales resultado de la producción y aplicación del abono a las áreas de cultivo.

\section{Materiales y métodos}

Por medio de herramientas metodológicas y experimentales se aplicó el abono orgánico denominado Supermagro al cultivo de Sandía (Citrullus lanatus) probados mediante 4 tratamientos, además se establecieron los impactos positivos y negativos, como los indicadores, y demás aspectos relevantes para dimensionar los efectos en la producción y utilización de abono orgánico Supermagro.

\section{1.Área de estudio}

La investigación se realizó en el Centro Experimental Universidad Surcolombiana, distrito de Riego Juncal municipio de Palermo en el departamento del Huila. El predio se ubica en las coordenadas $2^{\circ} 53^{\prime} 20.290^{\prime \prime}$ LN, 75 18' 37.227” LW y altitud de 442 m.s.n.m. Los re- 
gistros climatológicos para el periodo experimental se muestran en la Tabla 1.

Características del suelo. Se seleccionó la serieBosque (BS) cuyo relieve es ligeramente plano a partir del estudio detallado de suelos según la clasificación USDA 2006, donde se delimitaron siete series de suelos en el Centro Experimental La Universidad. La serie Bosque presenta las siguientes características físicas y químicas del suelo (Tabla 2).

Tabla 1. Registro Climatológico del Centro Experimental Universidad Surcolombiana.

\begin{tabular}{|c|c|c|c|c|c|c|c|c|c|}
\hline Año & Mes & $\begin{array}{c}\text { Temperatura } \\
\text { Media } \\
\left({ }^{\circ} \mathrm{C}\right) \\
\end{array}$ & $\begin{array}{c}\text { Humedad } \\
\text { Promedio } \\
(\%) \\
\end{array}$ & $\begin{array}{c}\text { Radiación } \\
\text { solar } \\
(\text { wat.m } \\
\text { (w) }\end{array}$ & $\begin{array}{c}\text { Velocidad } \\
\text { del viento } \\
\left(\mathrm{km} \cdot \mathrm{h}^{-1}\right)\end{array}$ & $\begin{array}{l}\text { Precipitación } \\
(\mathbf{m m})\end{array}$ & $\begin{array}{l}\text { Días } \\
\text { con } \\
\text { lluvia } \\
\end{array}$ & $\begin{array}{c}\text { Horas } \\
\text { Luz }\end{array}$ & $\begin{array}{l}\text { Evapo- } \\
\text { ración } \\
(\mathrm{mm})\end{array}$ \\
\hline 2013 & Sept & 26.3 & 58.1 & 371.6 & 15.7 & 35.03 & 6 & 172 & 148.3 \\
\hline 2013 & Oct & 25.2 & 70.0 & 352.3 & 7.8 & 101.25 & 16 & 276 & 215.9 \\
\hline 2013 & Nov & 23.7 & 85.2 & 326.0 & 5.7 & 215.91 & 18 & 239 & 176.5 \\
\hline 2013 & Dic & 24.1 & 83.9 & 329.9 & 4.5 & 131.15 & 13 & 258 & 193.2 \\
\hline 2014 & Ene & 24.9 & 77.4 & 356.1 & 5.7 & 86.48 & 9 & 260 & 210.1 \\
\hline 2014 & Feb & 25.3 & 76.8 & 358.4 & 6.3 & 80.45 & 11 & 239 & 191.1 \\
\hline 2014 & Mar & 23.1 & 89.5 & 282.7 & 5.5 & 68.01 & 5 & 31 & 26.3 \\
\hline \multicolumn{2}{|c|}{ Total } & 24.8 & 74.3 & 345 & 8.4 & 718.28 & 78 & 1475 & 1161.4 \\
\hline
\end{tabular}

Tabla 2. Propiedades químicas y físicas del suelo

\begin{tabular}{|c|c|c|c|c|c|c|c|}
\hline \multicolumn{4}{|c|}{ Propiedades químicas del suelo } & \multicolumn{4}{|c|}{ Propiedades físicas del suelo } \\
\hline Parámetros & Unidad & Resultado & Método & Parámetro & Unidad & Resultado & Método \\
\hline $\mathrm{pH}$ & - & 6.5 & NTC 5264 & $\mathrm{Da}$ & g. $\mathrm{cm}^{-3}$ & 1.35 & $\begin{array}{c}\text { Terrón } \\
\text { parafinado }\end{array}$ \\
\hline $\mathrm{CO}$ & $\%$ & 0.39 & $\begin{array}{l}\text { NTC } 5403- \\
\text { mét B }\end{array}$ & Dr & g. $\mathrm{cm}^{-3}$ & 2.60 & Picnómetro \\
\hline C.I.C. & $\mathrm{cmol}^{+} \cdot \mathrm{kg}^{-1}$ & 9.67 & NTC 5268 & $\mathrm{n}$ & $\%$ & 48.06 & $\begin{array}{c}\text { Rel. de } \\
\text { densidades }\end{array}$ \\
\hline $\mathrm{P}$ & ppm & 9.29 & NTC 5350 & DPM & $\mathrm{mm}$ & 1.51 & Yoder \\
\hline $\mathrm{Ca}$ & $\mathrm{cmol}^{+} \cdot \mathrm{kg}^{-1}$ & 5.89 & \multirow[t]{4}{*}{ NTC 5349} & $\mathrm{Ea}$ & $\%$ & 30.26 & Cálculo \\
\hline $\mathrm{Mg}$ & $\mathrm{cmol}^{+} \cdot \mathrm{kg}^{-1}$ & 2.16 & & $\mathrm{Pe}$ & $\mathrm{cm}$ & 22.0 & Campo \\
\hline $\mathrm{Na}$ & $\mathrm{cmol}^{+} \cdot \mathrm{kg}^{-1}$ & 0.30 & & AA. & $\%$ & 8.51 & Rel. CC y PMP \\
\hline $\mathrm{K}$ & $\mathrm{cmol}^{+} \cdot \mathrm{kg}^{-1}$ & 0.07 & & LAA & $\mathrm{cm} \cdot \mathrm{m}^{-1}$ & 11.49 & Cálculo \\
\hline BT & $\mathrm{cmol}^{+} \cdot \mathrm{kg}^{-1}$ & 8.42 & Suma cationes & $\mathrm{Ks}$ & $\mathrm{cm} \cdot \mathrm{h}^{-1}$ & 1.46 & Pozo barrenado \\
\hline SB & $\%$ & 87.07 & $\begin{array}{l}\text { Relación } \\
\text { catiónica }\end{array}$ & I & $\mathrm{cm} \cdot \mathrm{h}^{-1}$ & 5.43 & $\begin{array}{l}\text { Anillos } \\
\text { concéntricos }\end{array}$ \\
\hline $\mathrm{S}$ & ppm & 1.00 & NTC 5402 & \multirow[t]{4}{*}{$\begin{array}{l}\text { R.H } \\
\text { (bar) }\end{array}$} & $\begin{array}{l}0 \\
3 \\
3\end{array}$ & 13.18 & $\begin{array}{l}\text { Membranas de } \\
\text { Richards }\end{array}$ \\
\hline $\mathrm{Fe}$ & ppm & 70.0 & \multirow{3}{*}{$\begin{array}{l}\text { NTC } 5526 \\
\text { método DTPA }\end{array}$} & & $\%$ & 8.25 & \\
\hline $\mathrm{Mn}$ & ppm & 94.55 & & & $\%$ & 4.79 & \\
\hline $\mathrm{Cu}$ & ppm & 1.86 & & & $\begin{array}{l}1 \\
5\end{array}$ & 4.67 & \\
\hline B & ppm & 0.35 & $\mathrm{H}_{2} \mathrm{O}$ Caliente & Textura & clase & FA & Organoléptico \\
\hline $\begin{array}{l}\text { Acidez } \\
\text { intercambiable }\end{array}$ & $\mathrm{cmol}^{+} \cdot \mathrm{kg}^{-1}$ & - & NTC 5263 & \multicolumn{4}{|c|}{ FA Franco arenoso } \\
\hline $\begin{array}{l}\text { Relación } \\
\mathrm{Ca} / \mathrm{Mg}\end{array}$ & - & 2.73 & \multirow[t]{3}{*}{$\begin{array}{l}\text { Relación } \\
\text { catiónica }\end{array}$} & \multirow{3}{*}{\multicolumn{4}{|c|}{$\begin{array}{c}\text { Laboratorio de Suelos - LABGAA, Universidad } \\
\text { Surcolombiana. }\end{array}$}} \\
\hline $\begin{array}{l}\text { Relación }(\mathrm{Ca}+ \\
\mathrm{Mg}) / \mathrm{K}\end{array}$ & - & 115 & & & & & \\
\hline Relación $\mathrm{Mg} / \mathrm{K}$ & - & 30.86 & & & & & \\
\hline
\end{tabular}

Da es la densidad aparente, Dr es la densidad real, $\mathrm{n}$ es la porosidad total, DPM es el diámetro ponderado medio de partículas, R.H. en la retención de humedad gravimétrica a tensiones de $0.3,1,5$ y 15 bar, Ea es el espacio aéreo, Pe la profundidad efectiva del suelo, LAA es la lámina aprovechable del suelo, Ks es la conductividad hidráulica saturada e I es la infiltración básica del suelo. 
El suelo se localiza en clima calido - seco y se clasifica según USDA 2006 como Aridic Lithic Ustorthents escasamente desarrollado en profundidad y con limitaciones físico - químicas, ligeramente ácido y de baja fertilidad.

\subsection{Insumos}

Los insumos utilizados para la preparación del Supermagro fueron: 200 litros de agua (lluvia sin cloro), $60 \mathrm{~kg}$ de estiércol fresco, $12 \mathrm{~kg}$ de miel de purga o melaza, $1 \mathrm{~kg}$ de cal dolomita, $1 \mathrm{~kg}$ de sulfato de cobre, $1 \mathrm{~kg}$ de sulfato de magnesio, $1 \mathrm{~kg}$ de sulfato de zinc, 1 $\mathrm{kg}$ de bórax, $0.5 \mathrm{~kg}$ de sulfato de manganeso, $0.5 \mathrm{~kg}$ de sulfato de hierro, $1 \mathrm{~kg}$ de nitrato de potasio y 10 litros de suero homogenizados en una caneca de 200 litros de capacidad hasta completar el volumen total.

El Agrimins es un fertilizante mineral con fórmula reforzada de micronu-trientes y nutrientes secundarios complementado con nitrógeno y fósforo. Se debe aplicar como complemento de N, P, K, para hacer una fertili-zación completa, pero también se puede aplicar solo si el análisis de suelos así lo recomienda. Por poseer nitrógeno y fósforo se puede usar en ciertas condiciones como un abono compuesto; sin embargo, Agrimins es un complemento, no un sustituto, de los abonos tradicionales. Se aplica al suelo (siempre incorporado); ideal para complementar el abonamiento con fertili-zan-tes simples o compuestos. Se recomienda aplicar en suelos pobres o deficientes en calcio, magnesio, cobre, zinc, boro, molibdeno y azufre; para complementar también las aplicaciones de nitrógeno y fósforo.

\subsection{Metodología}

2.3.1 Preparación del abono "Supermagro". Es básicamente un aporte de elementos nutricionales ne- cesarios para el fortalecimiento y prevención de algunas enfermedades en los cultivos.

DÍA 1. Se preparan una caneca principal y 7 canecas complementarias. La caneca grande de 200 litros se completa con agua hasta la mitad, se agregan $60 \mathrm{~kg}$ de estiércol fresco, $3 \mathrm{~kg}$ de miel de purga disuelta en agua más 1 litro de leche, 1 kg de cal viva y se mezcla bien, se tapa herméticamente y se conecta con manguera para el escape del gas. En las 7 canecas pequeñas se preparan las mezclas dadas en la tabla 3.

DÍA 8. Después de un seguimiento diario, se agregan los siguientes productos: $0.5 \mathrm{~kg}$ de harina de huesos, $0.5 \mathrm{~kg}$ de harina de pescado más $0.5 \mathrm{~kg}$ de sangre de res y 0.5 kg de hígado licuado. Revolver cada día a la misma hora hasta el día 30.

DÍA 30. Está listo para ser utilizado. El indicador de terminación del proceso es cuando ha dejado de burbujear el gas de fermentación en el tubo de salida. El producto final es un líquido de color marrón, verdoso oscuro y de olor agradable. Se debe realizar un agujero en la tapa hermética de la caneca y conectar un tubo de escape de gases de fermentación, siempre dejar la mezcla tapada herméticamente. Para evacuar los gases producto de la fermentación, se debe pasar por la tapa un tubo conectado a una botella con agua para burbujeo. La manguera o tubo debe permanecer bajo el agua, el producto siempre se deja reposar a la sombra, se coloca antes de utilizar en la fumigadora o antes de envasar en otro recipiente oscuro. El preparado se recomienda utilizarlo en los 6 meses siguientes, aunque la experiencia ha mostrado que puede durar más tiempo (Garzón et al, 2014).

La fermentación dura aproximadamente 30 días, después el material debe ser colado para separar la

Tabla 3. Siete canecas de complemento Supermagro.

\begin{tabular}{|c|c|c|c|c|c|c|}
\hline \multicolumn{7}{|c|}{ Caneca } \\
\hline 1 & 2 & 3 & 4 & 5 & 6 & 7 \\
\hline $\begin{array}{l}1 \mathrm{~kg} \text { de } \\
\text { sulfato de } \\
\text { cobre, bien } \\
\text { molido } \\
\text { disuelto en } 3 \\
\text { litros de agua }\end{array}$ & $\begin{array}{l}1 \mathrm{~kg} \text { de sulfato } \\
\text { de magnesio, } \\
\text { disuelto en } 3 \\
\text { litros de agua }\end{array}$ & $\begin{array}{l}1 \mathrm{~kg} \text { de } \\
\text { sulfato de } \\
\text { zinc, disuelto } \\
\text { en } 3 \text { litros de } \\
\text { agua }\end{array}$ & $\begin{array}{l}1 \mathrm{~kg} \text { de } \\
\text { bórax } \\
\text { disuelto en } 3 \\
\text { litros de } \\
\text { agua }\end{array}$ & $\begin{array}{l}0.5 \mathrm{~kg} \text { de } \\
\text { sulfato de } \\
\text { manganeso } \\
\text { disuelto en } 3 \\
\text { litros de agua }\end{array}$ & $\begin{array}{l}0.5 \mathrm{~kg} \text { de } \\
\text { sulfato de } \\
\text { hierro disuelto } \\
\text { en } 3 \text { litros de } \\
\text { agua }\end{array}$ & $\begin{array}{l}1 \mathrm{~kg} \text { de sulfato } \\
\text { de potasio } \\
\text { disuelto en } 3 \\
\text { litros de agua }\end{array}$ \\
\hline \multicolumn{7}{|c|}{ Adicionar $1 \mathrm{~kg}$ de miel de purga disuelto en agua } \\
\hline \multicolumn{7}{|c|}{ Adicionar 1 litro de leche } \\
\hline \multicolumn{7}{|c|}{ Mezclar, revolver y tapar con un lienzo o plástico } \\
\hline
\end{tabular}


parte sólida más pesada de la parte líquida, que será utilizada como fertilizante orgánico en pulverizaciones foliares, sirviendo para la fertilidad de las plantas. Por otro lado, sirve para sustituir los fertilizantes químicos altamente solubles, los cuales son costosos y crean dependencia en los agricultores. Este producto no debe ser almacenado por mucho tiempo, para no alterar sus características (Santos, 1994).

2.3.2 Usos y dosis. Sirve para toda clase de cultivos, solo hay que ajustar la dosis. La dosis recomendada y la más usada es por cada 100 litros de agua, se mezclan 3 litros del caldo Supermagro, es decir 0.5 litro para una bomba de 20 litros (hasta 1 litro por bomba para árboles adultos frutales/café); al realizar la prueba de tolerancia para ajustar la dosis: mojar brotes tiernos y observar las reacciones de 1 día para el otro. En caso de reacciones negativas, bajar la dosis aumentando la cantidad de agua. Aplique el producto cuando el suelo esté húmedo (Nieto-Garibay, 2002).

2.3.3 Diseño experimental. Se utilizó diseño completamente al azar (DCA) con 4 tratamientos y 4 repeticiones. Se determinaron los grados de libertad de las fuentes de variación experimental, siendo los tratamientos de fertilización (supermagro 10\%, supermagro 30\%, Agrimins y el testigo) con 4 repeticiones en el cultivo de Sandía. El área total del ensayo fue de $348 \mathrm{~m}^{2}$ con 4 unidades experimentales de $30 \mathrm{~m}$ de largo por 0.90 $\mathrm{m}$ de ancho cada una, el número de plantas por unidad experimental fue de 150 plantas trasplantadas a una distancia de $20 \mathrm{~cm}$ entre ellas y $2 \mathrm{~m}$ entre camas para un total de 600 plantas (Tabla 4).

2.3.4 Variables e Indicadores. Para la medición de las variables de respuesta se asignaron indicadores, los cuales son descritos a continuación:

Altura de planta. La medición se realizó tomando una muestra al azar de 10 plantas establecidas dentro de la parcela útil, midiendo en cm desde la superficie del suelo hasta la base del ápice para cada tratamiento, cada 8 días después de su brote.
Diámetro del tallo. Los datos son tomados en mm de 10 tallos escogidos al azar por cada tratamiento, cada 8 días después de la germinación, en la parte media del tallo. La toma de los datos de los diámetros de los tallos, se tomó con un calibrador FWP Mauf, debidamente evaluado para establecer la precisión y validez de los registros.

Número de frutos por planta. Se realizó en 10 plantas de cada unidad experimental, de las mismas que se tomó para la altura de la planta y el diámetro del tallo, en este caso se obtuvo un promedio de cada parcela que se expresó para los frutos y datos registrados en unidades/planta (und/pl).

\subsubsection{Impacto Ambiental. Se utilizó la Matriz de} Leopold et al 1971, el primer paso consiste en identificar las iteraciones existentes tomando en cuenta todas las actividades del proyecto. Se recomienda operar con una matriz reducida, excluyendo las filas y las columnas que no tienen relación con el proyecto. Posteriormente y para cada acción, se considera todos los factores ambientales que puedan ser afectados significativamente, trazando una diagonal en las cuadriculas donde se interceptan con la acción. Cada cuadricula marcada con una diagonal admite dos valores:

Magnitud. Valoración del impacto o de la alteración potencial a ser provocada; grado, extensión o escala; se coloca en la mitad superior izquierda. Hace referencia a la intensidad, a la dimensión del impacto en sí mismo y se califica del 1 al 10 de menor a mayor, anteponiendo un signo $(+)$ para efectos positivos y $(-)$ para los negativos.

Importancia. Valor ponderal que da el peso relativo del potencial impacto, se escribe en la mitad inferior derecha del cuadro. Hace referencia a la relevancia del impacto sobre la calidad del medio, y la extensión o zona territorial afectada, se califica también del 1 al 10 en orden creciente de importancia. Una vez llenas las cuadricula el siguiente paso consiste en evaluar o

Tabla 4. Descripción de los tratamientos, productos y dosis de aplicación.

\begin{tabular}{|c|c|c|c|}
\hline Número de tratamiento & Código & Fertilizante & Dosis $\left(\mathrm{cm}^{3}\right) \mathrm{x}$ bomba (20 It) \\
\hline 1 & J1D1 & Supermagro $10 \%$ & 1000 \\
\hline 2 & $\mathrm{~J} 2 \mathrm{D} 2$ & Supermagro $30 \%$ & 3000 \\
\hline 3 & T.Q. & Testigo químico (Agrimins) & 1000 \\
\hline 4 & T.B. & Testigo en blanco (sin aplicación) & No se aplica \\
\hline
\end{tabular}

Agrimins es un producto comercial 
interpretar los números colocados (Matriz de valoración de impactos ambientales).

Calificación. La calificación se realiza así: Baja $=1$, Media $=2$ y Alta= 3; $1=$ Importancia del Impacto y 2= Magnitud del Impacto, para determinar la cuadricula:

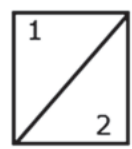

La agregación del impacto (AI), se obtiene multiplicando el valor de la magnitud con el de la importancia, de cada celda (todo horizontal o todo vertical) y los resultados se suman algebraicamente según cada columna o fila. Los valores que se registran en la AI, indican los beneficios o perjuicios de la acción propuesta.

\section{Resultados y discusión}

\subsection{Abono Supermagro}

El uso de este abono líquido foliar, permite nutrir la planta de forma orgánica con los elementos necesarios para su crecimiento vigoroso por ser rico en nutrientes. Al ser sana la planta, es más resistente al ataque de plagas y enfermedades, evitando la utilización de agro-tóxicos. La mayor concentración de los minerales se produce a los 30 días de fermentación, por lo que se recomienda utilizar este abono orgánico inmediatamente después de este tiempo.

\subsection{Variables evaluadas en el cultivo}

La toma y registros de las variables evaluadas en el cultivo de Sandía con los resultados obtenidos fueron:

Altura de planta (cm). La altura de la planta es un parámetro importante, ya que es un indicativo de la velocidad de crecimiento pero se puede ver afectada por la acción conjunta de los cuatros factores fundamentales: luz, calor, humedad y nutrientes. A su vez la altura está determinada por la elongación del tallo, al acumular es su interior los nutrientes producidos durante la fotosíntesis, los que a su vez son transferidos al fruto durante su desarrollo.

Por esta razón la toma de la altura de la planta se llevó a cabo muy minuciosamente, dejando bien especificada las plantas de sandía a evaluar y llevando un registro de principio a fin. La medida se tomó al tallo principal de cada planta, a su vez éste fue marcado para seguir con el protocolo de registrar una misma variable en tallo principal. Estadísticamente el crecimiento de las 10 plantas de sandía seleccionadas de las unidades experimentales, presentan diferencias significativas en cuanto a la elongación del tallo (Tabla 5).

El análisis de varianza indica que los tratamientos J1D2 al 30\% y el tratamiento químico (Agrimins) resultaron con mayor respuesta en crecimiento de las plantas de sandía, siendo significativamente superior (nivel de significancia del 5\%) con relación a los tratamientos J1D1 al 10\% y al testigo.

Tabla 5. Registro de altura de plantas de sandia.

\begin{tabular}{|c|c|c|c|c|c|c|c|c|c|c|c|c|}
\hline \multirow{4}{*}{$\begin{array}{c}\text { Número } \\
\text { de } \\
\text { planta }\end{array}$} & \multicolumn{12}{|c|}{ Altura de planta $(\mathrm{cm})$} \\
\hline & \multicolumn{3}{|c|}{$\begin{array}{c}\text { Tratamiento J1D1 } \\
10 \%\end{array}$} & \multicolumn{3}{|c|}{$\begin{array}{c}\text { Tratamiento J1D2 } \\
30 \%\end{array}$} & \multicolumn{3}{|c|}{$\begin{array}{c}\text { Tratamiento } \\
\text { Agrimins (T. Q) }\end{array}$} & \multicolumn{3}{|c|}{$\begin{array}{c}\text { Tratamiento en } \\
\text { blanco (T. B) }\end{array}$} \\
\hline & 20 & ( & 60 & 20 & 40 & 60 & 20 & 40 & 60 & 20 & $\overrightarrow{40}$ & 60 \\
\hline & & & & & & & & & Dias & & & 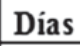 \\
\hline Sl & 35,30 & 52,40 & 60 & 60,30 & 102,80 & 30 & 30 & 30 & 120,40 & 23,20 & 43,40 & \\
\hline S2 & & & & & 60 & & & & & 70 & & \\
\hline S3 &, 20 & 56 , & 102,40 & 0,40 & 105,70 & 14 & 1,20 & 105,80 & 126,30 & 23,60 & 30 & - \\
\hline S4 & 0,20 & 62,90 & 50 & 1,60 & 108,40 & & 0,40 & 20 & 124,50 & 26,40 & & \\
\hline S5 & 8,80 & 54,20 & 96,90 & 61,50 & 107,60 & 15 & 60,80 & 93,50 & 138,90 & 23,20 & & - \\
\hline S6 & 7,20 & 51,80 &, 80 & 0,40 & 105,30 & 14 & 2,20 & 30 & 128,30 & 28,40 & & \\
\hline S7 & & & & & 106,40 & & 1,90 & 60 & 130,40 & 25,40 & & \\
\hline S8 & 36,30 & 55,80 & 30 & 1,10 & 110,10 & & 00 & & & 30,40 & & \\
\hline S9 & 8,90 & 57,50 & 40 & 60,70 & 107,20 & & 50 & & & 27,40 & 38,60 & 4,70 \\
\hline $\mathrm{S} 10$ & 20 & 56,70 & 108,40 & 61,30 & 108,60 & 15 & 61,90 & 93,80 & 140,80 & 25,60 & 43,80 & 5,50 \\
\hline$\dot{\mathbf{x}}$ & 37,73 & 56,98 & 101,68 & 60,93 & 107,27 & 149,47 & 61,74 & 98,89 & 130,49 & 25,63 & 43,69 & 88,85 \\
\hline 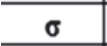 & & & & & & & & & & \begin{tabular}{|l|}
2,42 \\
\end{tabular} & & 2,10 \\
\hline C. & 5,31 & 6,55 & 3,25 & 0,74 & 2,07 & 0,52 & 1,37 & 5,12 & 4,53 & 9,45 & 6,91 & 2,37 \\
\hline
\end{tabular}


Tabla 6. Registro de diámetros de tallos en sandia.

\begin{tabular}{|c|c|c|c|c|c|c|c|c|c|c|c|c|}
\hline \multirow{4}{*}{$\begin{array}{c}\text { Número } \\
\text { de } \\
\text { planta }\end{array}$} & \multicolumn{12}{|c|}{ Diámetro de los tallos (mm) } \\
\hline & \multicolumn{3}{|c|}{$\begin{array}{c}\text { Tratamiento J1D1 } \\
10 \%\end{array}$} & \multicolumn{3}{|c|}{$\begin{array}{c}\text { Tratamiento J1D2 } \\
30 \%\end{array}$} & \multicolumn{3}{|c|}{$\begin{array}{c}\text { Tratamiento } \\
\text { Agrimins (T. Q) }\end{array}$} & \multicolumn{3}{|c|}{$\begin{array}{c}\text { Tratamiento en } \\
\text { blanco (T. B) }\end{array}$} \\
\hline & 20 & 10 & 60 & 20 & 40 & 60 & 20 & 40 & 60 & 20 & 40 & 60 \\
\hline & & & & Di & Dia & & & & & & & \\
\hline Sl & & & & 60,30 & 102,80 & & & & & & & \\
\hline $\mathrm{S} 2$ & & & 50 & 61 & 110,60 & & 0 & & & 70 & & 0,40 \\
\hline S3 & & 30 & 2,40 & 60,40 & 105,70 & 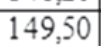 & 61,20 & 105,80 & 126,30 & 23,60 & & 0,40 \\
\hline S4 & & & 50 & 61,60 & 108,40 & & 0 & & 50 & 26,40 & 45,60 & 40 \\
\hline S5 & 80 & 54,20 & 96,90 & 61,50 & 107,60 & 150,20 & 60,80 & 0 & 138,90 & 23,20 & 39,80 &, 50 \\
\hline S6 & 20 & 80 & 80 & 60,40 & 105,30 & 149 & 62,20 & & 12 & 28,40 & 70 & 00 \\
\hline S7 & &, 60 & 90 & 60,80 & 106,40 & 148 & 6 & 10 & 130,40 & 25,40 & 44,50 & 60 \\
\hline S8 & 30 & 8,80 & 05,30 & 61,10 & 110,10 & 0 & 63,00 & 100,70 & 129,50 & 30,40 & 40,40 & 89,80 \\
\hline S9 & 90 & 57,50 & 99,40 & 60,70 & 107,20 & & 61,50 & & 132,60 & 27,40 & 38,60 & 84,70 \\
\hline $\mathrm{Sl0}$ & 20 & 6,70 & 8,40 & 61,30 & 108,60 & 150,20 & 61,90 & 93,80 & 140,80 & 25,60 & 43,80 & 85,50 \\
\hline$\dot{\theta}$ & 73 & 56,98 & 101,68 & 60,93 & 107,27 & 14 & 61,74 & 39 & 130,49 & 25,63 & 43,69 & 88,85 \\
\hline$\sigma$ & & 3,73 & & 0,45 & 2,22 & & & & 5,91 & 2,42 & 2 & 2,10 \\
\hline $\mathrm{Cr}$ & 5,31 & 6,55 & 3,25 & 0,74 & 2,07 & 0,52 & 1,37 & 5,12 & 4,53 & 9,45 & 6,91 & 2,37 \\
\hline
\end{tabular}

Diámetro de tallos (mm). La aplicación de nitrógeno es uno de los factores que incide en el diámetro de las plantas, alta dosis de nitrógeno influye positivamente es esta variable. La alta densidad de siembra y la competencia por luz con las malezas provocan una elongación de los tallos, entrenudos más largos y plantas más altas reduciendo el grosor de los tallos. Los tallos delgados es un símbolo de raquitismo por deficiencia del vegetal. El diámetro del tallo también es una característica de suma importancia en el cultivo y esta puede verse afectada por la densidad poblacional y el nitrógeno disponible. $\mathrm{La}$ variable diámetro de tallos en plantas de sandía, el análisis de varianza no presentó diferencias significativas al 5\%, para los tratamientos J1D1 al 10\%, J1D2 al 30\% y T.Q., evidenciándose diferencias significativas frente al testigo (Tabla 6).

Número de frutos por planta. Las unidades experimentales para el cultivo de sandía dieron sus frutos a los 50 días después de la siembra. El mejor tratamiento en producción de frutos resulto el J1D2 30\%, mediante el análisis de varianza con nivel del $5 \%$, frente a los tratamientos J1D1 10\% y T.Q. Agrimins, a su vez los tratamientos mencionados presentan diferencias significativas con relación al testigo (T.B.). Así mismo, se observa menor heterogeneidad en el tratamiento J1D2 30\% frente al resto de los tratamientos, dado por el coeficiente de variación (2.1.43\%) (Tabla 7).

\subsection{Evaluación del Impacto Ambiental Proyectado}

Para la evaluación del impacto ambiental por la matriz de Leopold, primero se identificaron los impactos, luego
Tabla 7. Registro de datos para el número de frutos.

\begin{tabular}{c|c|c|c|c}
\hline \multirow{2}{*}{\begin{tabular}{c} 
Número $\begin{array}{c}\text { Ne } \\
\text { planta }\end{array}$ \\
\cline { 2 - 5 }
\end{tabular}} & $\mathbf{J 1 D 1} \mathbf{1 0 \%}$ & $\mathbf{J 1 D 2} \mathbf{3 0 \%}$ & $\begin{array}{c}\text { Agrimins } \\
\text { (T.Q) }\end{array}$ & $\begin{array}{c}\text { Blanco } \\
\text { (T.B) }\end{array}$ \\
\cline { 2 - 5 } & $\mathbf{5 0}$ días & $\mathbf{5 0}$ días & $\mathbf{5 0}$ días & $\mathbf{5 0}$ días \\
\hline S1 & 1,00 & 3,00 & 3,00 & 1,00 \\
\hline S2 & 2,00 & 2,00 & 1,00 & 1,00 \\
\hline S3 & 1,00 & 3,00 & 2,00 & 0,00 \\
\hline S4 & 2,00 & 3,00 & 1,00 & 1,00 \\
\hline S5 & 2,00 & 2,00 & 2,00 & 1,00 \\
\hline S6 & 1,00 & 4,00 & 2,00 & 1,00 \\
\hline S7 & 3,00 & 3,00 & 1,00 & 1,00 \\
\hline S8 & 1,00 & 2,00 & 2,00 & 1,00 \\
\hline S9 & 2,00 & 3,00 & 2,00 & 1,00 \\
\hline S10 & 1,00 & 3,00 & 1,00 & 1,00 \\
\hline$\dot{\mathbf{x}}$ & $\mathbf{1 , 6 0}$ & $\mathbf{2 , 8 0}$ & $\mathbf{1 , 7 0}$ & $\mathbf{0 , 9 0}$ \\
\hline $\boldsymbol{\sigma}$ & $\mathbf{0 , 6 6}$ & $\mathbf{0 , 6 0}$ & $\mathbf{0 , 6 4}$ & $\mathbf{0 , 3 0}$ \\
\hline Cv & $\mathbf{4 1 , 4 6}$ & $\mathbf{2 1 , 4 3}$ & $\mathbf{3 7 , 6 7}$ & $\mathbf{3 3 , 3 3}$ \\
\hline
\end{tabular}

se procedió a la calificación y agregación de los impactos positivos y negativos de la matriz (Tabla 8).

La matriz de impactos de Leopold muestra que el mayor impacto cuantificado está relacionado con el componente biótico demostrado en los beneficios a la productividad del cultivo por aplicación del abono orgánico, seguido de la satisfacción socioeconómica y en la mejora de la calidad del producto.

\section{Conclusiones}

- La aplicación del abono denominado "Supermagro" al 30\% influye en las características agronómicas 
Tabla 8. Matriz de valoración de impactos ambientales.

\begin{tabular}{|c|c|c|c|c|c|c|c|c|c|c|c|c|c|c|c|c|c|}
\hline $\begin{array}{c}\text { Factores } \\
\text { ambientales }\end{array}$ & Elemento & 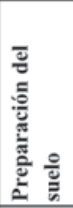 & 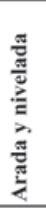 & 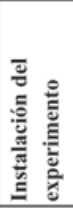 & 营 & 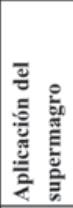 & 宽 & 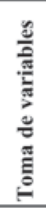 & 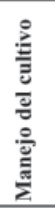 & 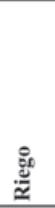 & 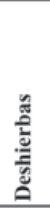 & 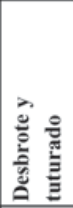 & 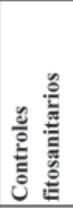 & 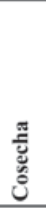 & 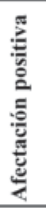 & 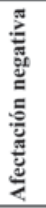 & 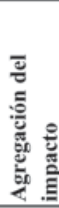 \\
\hline \multirow{8}{*}{ Abiótico } & Suelo & 3 & 2 & 1 & 3 & 3 & 3 & & 2 & 2 & 2 & & 2 & 2 & 6 & 5 & 10 \\
\hline & & -2 & -2 & 1 & 3 & 3 & -1 & & 2 & 1 & -1 & & -1 & 1 & & & \\
\hline & \begin{tabular}{|l|} 
Agua \\
\end{tabular} & 2 & & 2 & 3 & 3 & 1 & & & 2 & & & 2 & & 3 & 4 & 11 \\
\hline & & -2 & & 2 & 3 & 3 & -1 & & & -1 & & & -2 & & & & \\
\hline & Aire & 1 & & & & 2 & 2 & & & & & 2 & 2 & & 1 & 4 & -9 \\
\hline & & -1 & & & & -2 & -2 & & & & & 2 & -2 & & & & \\
\hline & \begin{tabular}{|l} 
Ambiente \\
\end{tabular} & 1 & & & & 2 & 2 & & & & & & 3 & & 0 & 4 & -15 \\
\hline & & -1 & & & & -2 & -2 & & & & & & -2 & & & & \\
\hline \multirow{12}{*}{ Biótico } & Flora & & 2 & & & 2 & 1 & & 2 & 2 & 2 & 2 & 2 & & 6 & 2 & 15 \\
\hline & & & 2 & & & 2 & -1 & & 2 & 1 & 1 & 2 & -2 & & & & \\
\hline & Fauna & & 2 & & & 2 & 1 & & & 2 & 2 & 1 & 2 & & 3 & 4 & -7 \\
\hline & & & -2 & & & -2 & -1 & & & 1 & 1 & 2 & -2 & & & & \\
\hline & Microflora & 3 & 2 & & 3 & 3 & 1 & & 2 & 2 & & & 2 & & 4 & 4 & 11 \\
\hline & & -2 & -2 & & 3 & 3 & -1 & & 2 & 2 & & & -2 & & & & \\
\hline & \begin{tabular}{|l|} 
Microfauna \\
\end{tabular} & 3 & 2 & & 3 & 3 & 1 & & 2 & 2 & & & 2 & & 4 & 4 & 11 \\
\hline & & -2 & -2 & & 3 & 3 & -1 & & 2 & 2 & & & -2 & & & & \\
\hline & Microorganismos & -3 & & & & 3 & & & 2 & 2 & & 2 & 2 & & 4 & 2 & 8 \\
\hline & Supermagro & 3 & & & & 3 & & & 2 & 2 & & 2 & -2 & & & & \\
\hline & \begin{tabular}{|l|} 
Cultivo \\
\end{tabular} & 3 & 2 & 3 & 3 & 3 & 3 & & 3 & 3 & 3 & 3 & 2 & 3 & 13 & 0 & 107 \\
\hline & & 3 & 2 & 3 & 3 & 3 & 3 & & 3 & 3 & 3 & 3 & 2 & 3 & & & \\
\hline \multirow{10}{*}{ Socio-económico } & Salud & & & & & 2 & 1 & & & & & 2 & 2 & & 2 & 2 & 1 \\
\hline & & & & & & 1 & -1 & & & & & 3 & -3 & & & & \\
\hline & Educación & 2 & 2 & 3 & 3 & 2 & 2 & & & & & & & & 7 & 0 & 45 \\
\hline & & 2 & 2 & 3 & 3 & 3 & 2 & & & & & & & & & & \\
\hline & Calidad de & 2 & 2 & 2 & 3 & 3 & 2 & & 2 & 2 & 3 & 3 & 2 & & 12 & 1 & 62 \\
\hline & \begin{tabular}{|l} 
producto \\
\end{tabular} & 2 & 2 & 2 & 3 & 3 & 2 & & 2 & 2 & 2 & 3 & -2 & & & & \\
\hline & \begin{tabular}{|l|} 
Ingresos \\
\end{tabular} & & & & & 3 & & & & & & 3 & 2 & 3 & 3 & 1 & 25 \\
\hline & & & & & & 3 & & & & & & 3 & -1 & 3 & & & \\
\hline & \begin{tabular}{|l} 
Satisfacción \\
\end{tabular} & 3 & 3 & 1 & 3 & 3 & 1 & & 2 & 2 & 2 & 3 & 2 & 3 & 13 & 0 & 76 \\
\hline & & 3 & 3 & 1 & 3 & 3 & 1 & & 2 & 2 & 2 & 3 & 2 & 3 & & & \\
\hline \multicolumn{2}{|c|}{ Afectaciones positivas } & 4 & 5 & 6 & 8 & 12 & 4 & & 4 & 8 & 5 & 9 & 2 & 4 & \multicolumn{3}{|c|}{ Comprobación } \\
\hline \multicolumn{2}{|c|}{ Afectaciones negtivas } & 7 & 4 & 0 & 0 & 3 & 9 & & 0 & 1 & 1 & 0 & 12 & 0 & & & \\
\hline \multicolumn{2}{|c|}{ Agregción personal } & -7 & 9 & 28 & 72 & 81 & 1 & & 31 & 33 & 21 & 56 & -40 & 29 & & & 351 \\
\hline
\end{tabular}

de altura de planta, engrosamiento del tallo y días de fructificación, demostrando su respuesta positiva en las diferentes etapas fenológicas del cultivo de sandía.

- El abono “Supermagro” demuestra ser un producto orgánico de fácil preparación y rico en nutrientes que evita la contaminación del medio ambiente y la dependencia a los agroquímicos.

- La producción de sandía con la aplicación del abono orgánico "Supermagro” resultó con impacto positivo, lo que incrementa los ingresos económicos y la satisfacción personal de los consumidores.

\section{Referencias bibliográficas}

1. Anacona Ch., Paula y Rojas G. Fayver J. 1999. Clasificación de los suelos de la Granja "La Universidad" con fines de riego y drenaje. Tesis de grado, Programa de Ingeniería Agrícola, Universidad Surcolombiana. Neiva. 100 p.
2. Claassen V.P., Carey J.L. 2004. Regeneration of nitrogen fertility in disturbed soils using composts. Compost Sci. \& Util 12(2):145-152.

3. García, I. y Dorronsoro, C. 1998. Contaminación del suelo. Contaminación por metales pesados. Disponible en: http://edafologia.ugr.es/conta/tema15/ riesgos.htm (Consultada el 12 de septiembre de 2014).

4. Garzón M., Leidy L. y Perdomo S., Fabián M. 2013. Evaluación de la influencia de biofertilizantes orgánicos en el crecimiento, desarrollo y rendimiento de cultivos hortofrutícolas en el ámbito de la seguridad alimentaria. Tesis de grado, Programa de Ingeniería Agrícola de la Universidad Surcolombiana. Neiva Huila. 96 p.

5. Gonzáles Huiman, F.S. 2011. Contaminación por fertilizantes: «Un serio problema ambiental». Artículo relacionado con el desarrollo rural y la agricultura sostenible. Disponible en URL: http:// fgonzalesh. blogspot.com/2011/01/contaminacion- 
por-fertilizantes-un.html. Consultado el 20 de septiembre de 2014).

6. Jaramillo J., D. F. 1983. Estudio detallado de suelos del lote "La Universidad”. Neiva: Informe Técnico, Programa de Ingeniería Agrícola, Universidad Surcolombiana. 27 p.

7. Leopold, L. B., F. E. Clarke, B. B. Hanshaw, and J. E. Balsley. 1971. A procedure for evaluating environmental impact. U.S. Geological Survey Circular 645, Washington, D.C.

8. Nieto-Garibay, A., Murillo-Amador B., TroyoDiéguez E., Larrinaga-Mayoral J.A. y García-Hernández J.L. 2002. El uso de compostas como alternativa ecológica para la producción sostenible del Chile (Capsicum annum L.) en zonas áridas. Interciencia. 27:417-421.
9. Santos R. H. S., V. W. D. Casali, A. R. Condé \& L. C. G. Miranda. 1994. Qualidade de alface cultivada com composto organico. Hort. Bras., Brasília, 12(1): 29-2.

10.USDA, 2006. Claves para la Taxonomía de Suelos, Departamento de Agricultura de los Estados Unidos. Servicio de Conservación de Recursos Naturales, Décima Edición, 339 p.

11. Valenzuela, L.M.; Días, V.T.; Osuna, R.J. 2012. Uso de abonos orgánicos en hortalizas. Facultad de agronomía. Universidad Autónoma de Sinaloa (UAS). Pág. 14-15. Disponible en URL: http://www.cultura organica.com/html/magnified.php?ID=27\&PAG=14 (consultada el 10 de agosto de 2014). 
